Pacific Journal of Mathematics

COUNTABLY GENERATED MODULES OVER COMMUTATIVE 


\title{
COUNTABLY GENERATED MODULES OVER COMMUTATIVE ARTINIAN RINGS
}

\author{
R. B. WARFIELD, JR.
}

A general method is given for constructing countably generated modules with a number of bizarre properties over any commutative Artinian ring which is not a principal ideal ring. The main result shows that if $R$ is a commutative local Artinian ring which is not a principal ideal ring, and the residue class field of $R$ is $k$, then any pathological property that holds for some $k[t]$-module also holds for a suitable $R$-module. This method gives easy and uniform proofs of many known results (and some new ones) concerning modules over these rings. A theorem of A. L. S Corner's, concerning countable endomorphism rings of torsion-free Abelian groups, is generalized to algebras over suitable discrete valuation rings, and applied to obtain further pathological results for modules over Artinian rings.

If $R$ is a commutative Artinian principal ideal ring, then all $R$-modules are direct sums of cyclic modules. If $R$ is a commutative Artinian ring which is not a principal ideal ring, then it is well known that $R$ has indecomposable finitely generated modules requiring arbitrarily many generators. (J. P. Jans assures me that this result was known for algebras in antiquity. For Artinian commutative rings, it is implicit in Colby [4], and explicit (in more general contexts) in Griffith [8] and Warfield [12].) Actually, if $R$ is not a principal ideal ring, $R$ has indecomposable modules which are not finitely generated [8], and, even, not countably generated, [13]. Recent work on noncommutative Artinian rings suggests that an equally sharp distinction may exist between rings of finite module type and other Artinian rings in general. ( $R$ has finite module type if there are only a finite number of isomorphism classes of finitely generated indecomposable modules.) We mention Roiter's theorem [11] that a finite dimensional algebra which is not of finite module type has indecomposable finitely generated modules requiring arbitrarily large numbers of generators, and Ringel and Tachikawa's theorem [10], that if $R$ has finite module type then every $R$-module is a direct sum of finitely generated indecomposable modules.

There is clearly a temptation to hope that even if a ring is not of finite module type, one still might be able to prove some general theorems about the good behavior of its infinitely generated modules. In the commutative case, we give a general method for 
disillusioning oneself of such hopes. As an application of the method, we prove the following theorem:

Theorem A. Let $R$ be a commutative Artinian ring which is not a principal ideal ring. Then

(i) $R$ has a countably generated indecomposable module which has a monic endomorphism which is not epic.

(ii) $R$ has a countably generated indecomposable module which has an epic endomorphism which is not monic,

(iii) $R$ has $2^{\kappa_{0}}$ nonisomorphic indecomposable countably generated modules,

(iv) $R$ has a set of three countably generated indecomposable modules, $A, B, C$, such that $B$ and $C$ are not isomorphic and such that $A \oplus B \cong A \oplus C$ (i.e. the Krull-Schmidt and cancellation properties fail).

(v) $R$ has a countably generated module with no indecomposable summands.

(vi) For any cardinal $n, R$ has a module which is not a summand of a direct sum of modules which can be generated by $n$ elements.

We recall that any commutative Artinian ring is a direct sum of local rings, so it is enough to prove our results for a local Artinian commutative ring which is not a principal ideal ring. If $R$ is such a ring, and $k$ is its residue class field, we will show that properties such as the above hold for modules over $R$ if they hold for modules over $k[t]$. Over the ring $k[t]$ of polynomials over a field $k$, examples of modules satisfying (i), (ii), (iii) and (vi) are quite elementary. This will give easy proofs of (i), (ii), (iii), (vi), and of some other results which are weaker than (iv) and (v). This also gives essentially trivial proofs of the existence of finitely generated indecomposable $R$-modules requiring arbitrarily many generators, indecomposable $R$-modules which are countably, but not finitely, generated, and indecomposable $\boldsymbol{R}$-modules requiring an uncountable number of generators. (There last results were previously known, and are in the above references. (vi) was proved in [13].)

The results (iv), and (v) are more difficult, and require an extension of methods of A.L.S. Corner's, first used to prove similar results for Abelian groups. A somewhat different adaptation of Corner's methods is used by Brenner in [3], to prove these same results for a large class of rings, including many noncommutative rings, but not including all commutative Artinian artinian rings which are not principal ideal rings. In particular, Brenner's results in [2], on group rings, omit the group $(Z / 2 Z) \oplus(Z / 2 Z)$, which is not in any way excluded by our 
methods (i.e. if $k$ is a field of characteristic $2, k[(Z / 2 Z) \oplus(Z / 2 Z)]$ is a ring for which our results hold.)

There are a number of other pathological results known for Abelian groups that one might expect to generalize to modules over the rings $k[t]$, and, therefore, (by our results) to modules over commutative Artinian rings which are not principal ideal rings. We refer to Fuchs [7, $\S \S 88-91]$, and the references there, for some such results, some of which have recently been extended to large cardinals by Shelah (not yet published). The proofs of such pathological results tend to depend on various arithmetic properties of the ring of integers, and, hence, tend not to generalize immediately to the rings $k[t]$. It is probably true, however, that with some ingenuity, most of these results can be extended, if one happens to wish to do so.

1. Translatable modules over an Artinian ring. We recall (e.g. from [13, Lemma 1]) that if $R$ is a commutative Artinian ring which is not a principal ideal ring, then $R$ contains an ideal $I$ such that $R / I$ is a local ring with maximal ideal $m$, such that $m^{2}=0$ and $m$ has $R / m$-dimension 2 . We may assume, therefore, that $R$ is originally a ring of this sort, and that $m$ is generated by the two elements $a$ and $b$. An example of such a ring is $k[x, y] /\left(x^{2}, x y, y^{2}\right)$. We let $k=R / m$.

If $M$ is any $R$-module, multiplication by $a$ and $b$ respectively induce $k$-linear transformations $\alpha$ and $\beta$, from $M / m M$ to $m M$. We will say that $M$ is a-translatable if $\alpha$ is an isomorphism of $M / m M$ onto $m M$.

If $M$ is a-translatable, we define a translation (linear transformation) of $M / m M$ as follows: if $x \in M / m M$, there is a unique $y \in M / m M$ such that $\alpha(y)=\beta(x)$. Let $t x=y$. This defines a linear transformation of $M / m M$ into itself, and gives $M / m M$ a natural $k[t]$-module structure.

We note that if $M$ and $N$ are $a$-translatable $R$-modules, and $f: M \rightarrow N$ is a homomorphism, then $f$ induces a $k[t]$-module homomorphism $M / m M \rightarrow N / m N$. $(f(M)$, however, need not be an $a$-translatable submodule of $N$.) We have therefore defined a functor from the category of $a$-translatable $R$-modules (a full subcategory of the category of all $R$-modules) to the category of $k[t]$-modules.

THEOREM B. Let $\Phi$ be the functor associating to each atranslatable module $M$, the $k[t]-$ module $M / m M$. Then for any $k[t]$ module $N$, there is an a-translatable $R$-module $M$ such that $\Phi(M) \cong$ $N$. Further, if $A$ and $B$ are $k[t]$-modules, and $f: A \rightarrow B$ a homomorphism, and if $M$ and $N$ are a-translatable $R$-modules such that $\Phi(M)=$ $A, \Phi(N)=B$, then there is an $R$-homomorphism $g: M \rightarrow N$ such that $\Phi(g)=f$. If $f$ is monic, so is $g$, and if $f$ is epic, so is $g$. If $\Phi(M)=A$, 
then any direct sum decomposition of $M$ gives rise to a decomposition of $A$, and any decomposition of $A$ arises from a decomposition of $M$.

This theorem is proved by a series of lemmas. We first write down an example of an $a$-translatable module. Let $M$ be the module defined by taking elements $x_{i}(1 \leqq i<\infty)$ as generators, and imposing the relations $b x_{i}=a x_{i+1}(1 \leqq i<\infty) . \quad M$ is clearly a-translatable, and $\Phi(M)=$ $k[t]$ (regarded as a module over itself). Taking sums of copies of this $M$, we see that any free $k[t]$-module is a value of the functor $\Phi$. To go further, we naturally consider $a$-translatable submodules of atranslatable modules.

Lemma 1. If $M$ is a-translatable, and $K$ a submodule, then $K$ is a-translatable if and only if for every $x \in M$ such that ax $\in K, x \in m M+$ $K$. In this case, aK $=K \cap m M, K / m K$ is a $k[t]$-submodule of $M / m M$, and $M / K$ is a-translatable.

Proof. If $K$ is a-translatable then $m K=a K$. Also, if $y \in K$ and $a y=0$, then $y \in m K$. Hence, $m K=a K=K \cap m M$. Also, if $x \in M$, and $a x \in K$, then $a x \in a K$, whence $x \in K+m M$. To show the converse, suppose that $K$ is a submodule of $M$ with the property that if $x \in M$ and $a x \in K$, then $x \in m M+K$. If $z \in K \cap m M, z=a x$ for some $x \in M$, so for some $x^{\prime} \in m M, x-x^{\prime} \in K$. Clearly, $a\left(x-x^{\prime}\right)=$ $a x$, so $z \in a K$. This shows that $K \cap m M=a K$. The map $\alpha: K / m K \rightarrow m K$ is therefore onto, and it is also one-to-one, since $x \in K, x \notin m K$ implies $x \notin m M$ (since $K \cap m M=a K$ ), so $a x \neq 0$.

It is clear that if $K$ is an $a$-translatable submodule of $M$, then $K / m K$ can be regarded as a $k[t]$-submodule of $M / m M$, so all that remains is to show that $M / K$ is also $a$-translatable.

If $x \in M / K$, $a x=0$, and $x=y+K$, then $a y \in K$, so $y \in m M+K$, which implies that $x \in m(M / K)$. To show that $M / K$ is $a$-translatable, therefore, we need only show that $m(M / K)=a(M / K)$, which follows from the fact that $m(M / K)=(m M+K) / K=(a M+K) / K$.

LemMA 2. If $M$ is a-translatable, and $L$ a $k[t]$-submodule of $M / m M$, then there is an a-translatable submodule $K$ of $M$ such that $K / m K=L$.

Proof. Choose a set $X$ in $M$ such that the natural map $\phi: M \rightarrow M / m M$ takes $X$ bijectively onto a basis for $M / m M$ as a $k$-vector space, and such that $\phi(X) \cap L$ is a basis for $L$. Let $K$ be the submodule of $M$ generated by those $x \in X$ such that $\phi(x) \in$ $L$. Clearly $K /(K \cap m M)=L$. Suppose $x \in M$ and $a x \in K$. It is clear by construction that $m K=K \cap m M$, so $a x=a y+b z$, for suitable 
$y$ and $z$ in $K$. If we choose $w$ such that $a w=b z(w \in M)$ then $t(z+m M)=w+m M$, and, since $L$ is a $k[t]$-submodule of $M / m M$, there is a $w^{\prime} \in K$ and a $w^{\prime \prime} \in m M$ such that $w=w^{\prime}+w^{\prime \prime}$. Hence $b z=a w=a w^{\prime} \in a K$, so $a x=a\left(y+w^{\prime}\right)$. Since $M$ is a-translatable, this means $x-y-w^{\prime} \in m M$, so $x \in K+m M$ as required.

LeMma 3. If $M$ is a-translatable, and $M / m M=\bigoplus_{i \in I} A_{i}$, where the decomposition is a $k[t]$-module decomposition, then $M=\bigoplus_{i \in I} B_{i}$ where the $B_{i}$ are a-translatable submodules, and $\left(B_{i}+m M\right) / m M=A_{i}$ for all $i \in I$.

Proof. Choose a subset $X$ of $M$ such that the natural map $\phi: M \rightarrow M / m M$ takes $X$ bijectively onto a basis of $M / m M$, with the property that $\phi(X) \cap A_{i}$ is a basis for $A_{i}$ (as a $k$-vector-space) for all $i \in I$. Let $B_{i}$ be the $R$-submodule of $M$ generated by those $x \in X$ such that $\phi(x) \in A_{i}$. By the previous lemma, the modules $B_{i}$ are $a$ translatable. By construction, $B_{j} \cap \sum_{i \neq j} B_{i} \subseteq m M$, and, by the argument of the previous lemma, $B_{j} \cap m M=a B_{j}=\alpha\left(A_{j}\right)$, so the sum of the $B_{i}$ is a direct sum. If $B=\bigoplus_{i \in I} B_{i}$, then $M=B$, since, by construction, $M=B+m M$, and $m M \subseteq B$, since $m M=m B+m^{2} M=m B$.

LEMMA 4. If $M$ and $N$ are a-translatable $R$-modules and $f: M / m M \rightarrow N / m N$ a $k[t]$-module homomorphism, then there is an $R$-homomorphism $g: M \rightarrow N$ such that $g$ induces the map $f . \quad$ Further, if $f$ is a monomorphism, then any such $g$ is a monomorphism, and if $f$ is an epimorphism, then any such $g$ is an epimorphism. In particular, if $f$ is an isomorphism, so is $g$.

Proof. Choose subsets $X$ and $Y$ of $M$ and $N$ such that if $\phi: M / m M$ and $\psi: N \rightarrow N / m N$ are the natural maps, then $\phi$ and $\psi$ take $X$ and $Y$ bijectively onto $k$-bases of $M / m M$ and $N / m M, \phi(X) \cap$ $\operatorname{Ker}(f)$ spans $\operatorname{Ker}(f)$ over $k$, and $f \phi(X) \subseteq \psi(Y)$. If $x \in X$, define $g(x)=y$, where $y \in Y$ and $y$ is chosen so that $\psi(y)=f \phi(x)$.

We would like to extend $g$ to $M$ as a module homomorphism (which can be done in at most one way, since $X$ generates $M$ over $R$ ). To show this is possible, we need to show that if $\left\{x_{i}: i=1, \cdots, n\right\}$ are distinct elements of $X$, and $y_{i}=g\left(x_{i}\right)$, and there are elements $r_{i}$ and $s_{i}$ in $R$ such that

$$
r_{1} x_{1}+\cdots+r_{n} x_{n}=s_{1} x_{1}+\cdots+s_{n} x_{n}
$$

then, also

$$
r_{1} y_{1}+\cdots+r_{n} y_{n}=s_{1} y_{1}+\cdots+s_{n} y_{n}
$$


It suffices to consider the special case $r_{1} x_{1}+\cdots+r_{n} x_{n}=0$, with the $x_{i}$ distinct, which clearly implies that $r_{i} \in m$ for all $i$. We write $r_{i}=$ $a u_{i}-b v_{i}$. The equation then can be written

$$
\alpha\left(u_{1} \phi\left(x_{1}\right)+\cdots+u_{n} \phi\left(x_{n}\right)\right)=\beta\left(v_{1} \phi\left(x_{1}\right)+\cdots+v_{n} \phi\left(x_{n}\right)\right) .
$$

We want this to imply that

$$
\alpha\left(u_{1} \psi\left(y_{1}\right)+\cdots+u_{n} \psi\left(y_{n}\right)\right)=\beta\left(v_{1} \psi\left(y_{1}\right)+\cdots+v_{n} \psi\left(y_{n}\right)\right) .
$$

Since $\psi\left(y_{1}\right)=f \phi\left(x_{i}\right)$, if $x=v_{1} x_{1}+\cdots+v_{n} x_{n}$, and $z=u_{1} x_{1}+\cdots+u_{n} x_{n}$, then the first equation says $z=t x$, and the second says $f(z)=$ $t f(x)$. The first, therefore, implies the second, since $f$ is a $k[t]-$ homomorphism.

Suppose, now, that $f$ is a monomorphism. Since $a M$ is essential in $M$, it is enough to show that $\operatorname{Ker}(g) \cap a M=0$. On $a M$, an easy computation shows that $g=\alpha f \alpha^{-1}$, a composite of monomorphisms, which proves the result. If $f$ is an epimorphism, then $N=g(M)+m N$, and $m N \subseteq g(M)$, since $m N=m g(M)+m^{2} N$ and $m^{2}=0$.

These various lemmas prove Theorem B. In particular, we know that for any free module $F$, there is an $a$-translatable module $M$ such that $\Phi(M)=F$. By Lemma 2, if $L$ is a submodule of $F$, there is an a-translatable submodule $K$ of $M$ such that $\Phi(K)=L$. In this case, Lemma 1 implies that $M / K$ is an $a$-translatable module, and $\Phi(M / K)=$ $F / L$. Hence, every $k[t]$-module is of the form $\Phi(N)$. The remainder of the theorem is contained in Lemmas 3 and 4.

2. Elementary applications of Theorem B. In this section, we write down some completely elementary facts about modules over the ring $k[t]$, and derive examples by using Theorem $\mathrm{B}$. We give elementary proofs of the following facts: If $R$ is a commutative Artinian ring which is not a principal ideal ring, then

(a) $R$ has finitely generated indecomposable modules requiring arbitrarily large numbers of generators,

(b) $R$ has a countably generated indecomposable module which has a monic epimorphism which is not epic,

(c) $R$ has a countably generated indecomposable module which has an epic endomorphism which is not monic,

(d) $R$ has a set of $2^{x_{0}}$ nonisomorphic indecomposable countably generated modules,

(e) $R$ has a countably generated module with two direct sum decompositions which do not have isomorphic refinements,

(f) $R$ has a countably generated module which is not a direct sum of indecomposable modules, 
(g) For any cardinal $n, R$ has a module requiring more than $n$ generators, all of whose direct sum decompositions are finite.

In particular, when this has been done, we will have proved parts (i), (ii), and (iii) of Theorem A. Also, ( $g$ ) easily implies (vi), since, by the higher cardinal form of Kaplansky's lemma [9], a summand of a direct sum of modules with $n$ generators is again a direct sum of modules with $n$ generators, for any infinite cardinal $n$.

We first note that $k[t]$ as a module over itself is an indecomposable module of countably infinite $k$-dimension, which admits a monic endomorphism which is not epic. This example proves (b). (We notice that if $\Phi(M)=A$, then the nunber of generators required for $M$ is the $k$-dimension of $A$.) If $M_{n}$ is an $a$-translatable $R$-module such that $\Phi\left(M_{n}\right)=k[t] /\left(t^{n}\right), M_{n}$ is an indecomposable $R$-module requiring exactly $n$ generators. This proves (a).

The set $N$ of $k$-rational functions in $t$ of the form $t^{n} f$, where $f \in k[t]$ and $n$ is any integer, is a $k[t]$-submodule of the quotient field of $k[t] . \quad N / k[t]$ is an indecomposable $k[t]$-module with the descending chain condition which admits an epic endomorphism which is not monic. (This is the analogue over $k[t]$ of the Abelian group $Z\left(p^{\infty}\right)$, [7, vol. I, p. 15].) This proves (c).

Choose a countably infinite set $C$ of inequivalent irreducible nonconstant polynomials in $k[t]$. For every subset $D$ of $C$, let $M(D)$ be the set of rational functions of the form $g / f$, where $g \in k[t]$ and $f$ is an element of $k[t]$ all of whose irreducible factors are in the set $D$. The modules $M(D)$ are all indecomposable torsion-free $k[t]$-modules of rank one, and all countably generated. They are pairwise nonisomorphic. (See [7, sections 85 and 86] for the Abelian group analogue.) This proves (d).

An indecomposable $k[t]$-module is either torsion or torsion-free, and the indecomposable torsion modules are either divisible or cyclic [7, 3.1 and 27.4]. Following Prüfer, (or [7, I, p. 150]), we consider the torsion module $M$ given by generators and relations as follows: the generators are $x_{i}, i=0,1, \cdots, n, \cdots$, and the relations $t x_{0}=0, t^{n} x_{n}=$ $x_{0}(n>0) . \quad M$ is clearly not a direct sum of indecomposable modules, which proves (f). In any decomposition of $M, M=B \oplus C$, one of the summands is a direct sum of cyclic modules while the other is not. Using Ulm's theorem, it is easy to show (Baer, [1]) that $M$ has two direct sum decompositions which do not have isomorphic refinements. This proves (e).

For any index set $I$, the $k[t]$-module $\Pi_{i \in I} k[[t]]$ is a module all of whose direct sum decompositions have only a finite number of summands, [13]. This proves (g). 
3. An adaptation of Corner's theorem. We prove the following theorem:

THEOREM C. Let $R$ be a discrete valuation ring, $R *$ its completion, and suppose that $R^{*}$ has uncountable transcendence degree over $R$. Then if $A$ is any countably generated $R$-algebra which is torsion-free and reduced (no injective summands) as an $R$-module, there is a countably generated torsion-free $R$-module $M$ such that $\operatorname{End}(M) \cong A$.

The proof is a straightforward application of the method used to prove the corresponding result for Abelian groups in [5]. The details appear later in this section. We first give the applications to modules over commutative Artinian rings. To show that Theorem $\mathrm{C}$ is applicable, we need the following lemma.

LEMMA 5. If $k$ is a field, the power series ring $k[[t]]$ has transcendence degree at least $2^{\boldsymbol{N}_{0}}$ over $k$.

Proof. We first note that if $k$ is a prime field $(Z / p Z$ or the field of rational numbers), then $k[[t]]$ has cardinality $2^{\kappa_{0}}$, while any countably generated extension of $k$ is countable, so the result is clear for such fields. We now let $K$ be an arbitrary field, and $k$ a prime field in $K$, and regard $k[[t]]$ as a subring of $K[[t]]$. We claim that if $p_{i}(i \in I)$ are elements of $k[[t]]$ which are algebraically independent over $k$, then, as elements of $K[[t]]$, they are algebraically independent over $K$. Suppose, then, that there were elements $p_{i}(i=1, \cdots, n)$ algebraically independent over $k$, and an equation $\Sigma r_{a} p^{a}=0$, where the index $a$ ranges over a finite set of lattice points $\left(a_{1}, \cdots, a_{n}\right), a_{i} \geqq 0$, and $p^{a}=$ $p_{1}^{a_{1}} \cdots p_{n}^{a_{n}}$. For every $a, p^{a}$ is a power series over $k$ with coefficients $q(a, i), 0 \leqq i<\infty$, and the equation would say $\Sigma r_{a} q(a, i)=0$ for all $i$. We regard these equations as a set of equations in the variables $r_{a}$, with coefficients $q(a, i)$. Our hypothesis says that these equations do not have a simultaneous solution over the field $k$. An infinite set of linear equations in $m$ variables ( $m$ constant) has a solution if and only if every finite subset has a solution, and a finite subset has a solution in $K$ if and only if it has a solution in $k$, so the insolubility of these equations over $k$ implies their insolubility over $K$. This implies that the elements $p_{i}(i=1, \cdots, n)$ remain algebraically independent over $K$, as desired.

To prove parts (iv) and (v) of Theorem A, it suffices to prove them for modules over the ring $k[t]$, (by Theorem B). For each of these properties, it is possible to write down a property of a ring $E$ such that if End $(M)=E$, then $M$ has the indicated property. In [5, pp. 708-9], Corner constructs an algebra $E$ over the ring of integers such that for any nonzero idempotent $\alpha$ of $E$, there is a nonzero idempotent $\beta$ of $E$, 
distinct from $\alpha$, such that $\alpha \beta=\beta$. His construction is completely valid over any commutative integral domain. In particular, we let $R=$ $k[t]_{(t)}$, the localization of $k[t]$ at the prime ideal $(t)$, and let $E$ be an $R$-algebra constructed in this way. By Theorem C, there is a countably generated $R$-module $N$ with $E$ as its endomorphism ring. If $R$ is countably generated as a $k[t]$-module (which is true if $k$ is countable), then $N$ is already a module of our desired sort, and the proof of $(v)$ is completed by an application of Theorem B. If $R$ is not countably generated over $k[t]$, we can construct a $k[t]$-submodule of $N$ with the desired properties. We note that from Corner's construction, $E$ only has a countable number of idempotents. We let $Y_{1}$ be a countable subset of $N$ that generates $N$ over $R$. Define $Y_{n+1}$ inductively to the set of elements of $N$ of the form $\alpha x$, where $x \in Y_{n}$ and $\alpha$ is an idempotent of $E$. Let $Y$ be the ascending union of the sets $Y_{n}$, and let $M$ be the $k[t]$-submodule of $N$ generated by $Y . M$ is clearly countably generated over $k[t]$, and, by construction, it has no indecomposable summands. Applying Theorem B, we now obtain a proof of (v) in complete generality.

The proof of (iv) involves a minor adaptation of a construction given by Corner and Crawley in [6]. Again, by Theorem B, it is enough to prove that the pathology can occur for modules over the ring $k[t]$. We first let $R=k[t]_{(t)}$ as before, and construct an algebra $S$ over $R$. Let $S$ be the ring of matrices of the form

$$
\left(\begin{array}{ll}
f_{11} & \left(1-s^{2}\right) f_{12} \\
f_{21} & a+\left(1-s^{2}\right) f_{22}
\end{array}\right)
$$

where the $f_{i j}$ are elements of the ring $R[s]$, and $a \in R . \quad S$ is clearly a countably generated algebra over $R$. This ring has idempotents $\alpha$ and $\beta$ such that $\alpha$ and $\beta$ are equivalent, but $1-\alpha$ and $1-\beta$ are not equivalent, and such that if $M$ is an a $R$-module with $S$ as endomorphism ring, then $\alpha M, \beta M,(1-\alpha) M$, and $(1-\beta) M$ are all indecomposable. The proof of these facts is contained in [6], though there is a possibility of confusion arising from the fact that in that paper, $S$ is only a subring of the endomorphism of an Abelian p-group, and there are a number of other things that the authors have to keep track of in the course of their argument. Briefly, one defines

$$
\theta=\left(\begin{array}{cc}
s & 1-s^{2} \\
0 & 0
\end{array}\right) \quad \phi=\left(\begin{array}{ll}
s & 0 \\
1 & 0
\end{array}\right)
$$

and $\alpha=\theta \phi, \beta=\phi \theta$. Noting that $\theta \phi \theta=\theta$ and $\phi \theta \phi=\phi$, we see that $\alpha$ and $\beta$ are equivalent idempotents. If $1-\alpha$ and $1-\beta$ were equivalent, then there would be matrices in $S, \mu$ and $\nu$, such that $\mu \nu=1-\alpha$, 
$\nu \mu=1-\beta, \mu \nu \mu=\mu$, and $\nu \mu \nu=\nu$. Ignoring the statement about reducing modulo $p$ in [6], the last paragraph in [6] provides a proof of the impossibility of the existence of $\mu$ and $\nu$. Hence, if $M$ is an $R$-module with $S$ as its endormorphism ring, we have $M=\alpha M \oplus(1-\alpha) M=$ $\beta M \oplus(1-\beta) M$, with $\alpha M \cong \beta M,(1-\alpha) M \not \equiv(1-\beta) M$. The summands appearing here are all indecomposable, since $S$ is a subring of a two-by-two matrix ring over a field, so that there cannot be a set of three orthogonal idempotents in $S$.

This establishes all that is needed (using Theorem B) to prove (iv), except for the fact that if $R$ is not countably generated over $k[t]$, then $M$ will not be countably generated. In this case, as before, we construct a countably generated $k[t]$-submodule of $M$ which still has the same pathology, using exactly the same technique as before (which is easier in this case, since we do not need to consider all idempotents of $S$, but only the idempotents $1, \alpha$, and $\beta$.)

We now prove Theorem C. Let $R$ be a discrete valuation ring, $R^{*}$ its completion, $p$ a prime element, and we assume that the quotient field of $R^{*}$ has uncountable transcendence degree over the quotient field of $R$. We recall that if $Q$ is the quotient field of $R$, then $Q \otimes_{R} R^{*}$ is the quotient field of $R^{*}$.

Let $A$ be a countably generated, reduced, torsion-free $R$-algebra, and let $X$ be a maximal $R$-independent subset of $A$, such that $1 \in$ $X$. We must find a countably generated, torsion-free module $M$ whose endomorphism ring $E(M)$ is isomorphic with $A$.

The $p$-adic completion $A^{*}$ of $A$ is a torsion-free $R^{*}$-module. We regard $A$ as an $R$-submodule of $A^{*}$, and choose a family $f_{i}(i \in I)$ of elements of $A$ which is a maximal set of elements which are independent over. $R^{*}$. For any $x \in X$, there are elements $r(x), r(x, i)(i \in I)$ of $R^{*}$ such that

$$
r(x) x+\sum_{i \in I} r(x, i) f_{i}=0,
$$

where $r(x, i)=0$ for all but a finite number of indices $i$. Multiplying by a suitable unit of $R^{*}$, we obtain

$$
p^{k} x=\sum s(x, i) f_{i}
$$

where the elements $s(x, i)$ are determined uniquely up to a factor of a power of $p$. Let $S$ be the pure $R$-subalgebra of $R^{*}$ generated by the elements $s(x, i)(x \in X, i \in I) . \quad S$ is a countably generated $R$-algebra, from which it follows that the transcendence degree of $R^{*}$ over $S$ is uncountable.

Lemma 6. If $\Sigma_{j \in J} g_{j} a_{j}=0$, where $g_{j} \in R^{*}$, the elements $g_{j}$ are independent over $S, a_{j} \in A$, and $J$ is a finite set, then the $a_{j}$ are all zero. 
Proof. For some fixed $n, p^{n} a_{j}=\sum r(x, j) x(x \in X, r(x, j) \in R)$.

We recall that there is a fixed $k$ such that for all $x$ (a finite number) that appear in the above equations,

$$
p^{k} x=\Sigma s(i, x) f_{i} ; \quad s(i, x) \in S
$$

The equation becomes

$$
\sum g_{j} r(x, j) s(i, x) f_{i}=0
$$

(the summation being over $j, x$, and $i$-the Einstein convention). Since the $f_{i}$ are independent over $R^{*}$, we obtain

$$
\sum g_{j} r(x, j) s(i, x)=0
$$

(summed over $j$ and $x$.) Since the $g_{j}$ are independent over $S$,

$$
\sum r(x, j) s(i, x)=0
$$

for each $i$ and $j$. Since

$$
p^{n+k} a_{j}=\sum_{i}\left(\sum_{x} r(x, j) s(i, x) f_{i}\right)
$$

and $A$ is torsion-free, we conclude that $a_{j}=0$, for all $j$.

We now return to the proof of the theorem. Choose elements $a(x), b(x)(x \in X)$ of $R^{*}$ that are algebraically independent over $S$, and define elements $e(x)(x \in X)$ of $A^{*}$ by setting

$$
e(x)=a(x) \cdot 1+b(x) \cdot x .
$$

Let $M$ be the pure $R$-submodule of $A^{*}$ generated by $A$ and $e(x) A$, $x \in X . \quad M$ is clearly countably generated, reduced, and torsion-free over $R$.

Since $A^{2} \subseteq A$, it is clear that $M A \subseteq M$, so $A$ operates on the right of $M$. Since $1 \in M$, the map $A \rightarrow \operatorname{End}(M)$ obtained in this way is one-to-one. To show that this map is an isomorphism, we must show that every endomorphism of $M$ coincides with right multiplication by some element of $A$.

If $\theta$ is an endomorphism of $M$ (acting on the right), then $\theta$ extends to an $R^{*}$-endomorphism $\theta^{*}$ of $A^{*}$, (since $\left.A^{*}=M^{*}\right)$. If $x \in X$, then

$$
e(x) \theta=(a(x)) \cdot 1) \theta^{*}+(b(x) \cdot x) \theta^{*}=a(x)(1 \cdot \theta)+b(x)(x \theta) .
$$


Since $1 \theta, e(x) \theta$, and $x \theta$ are all in $M$, we can write

$$
\begin{gathered}
p^{k}(1 \theta)=\sum_{y \in X} r_{1}(y) y+\sum_{\substack{w \in X \\
z \in X}} t_{1}(z, w) e(z) w \\
p^{k} e(x) \theta=\sum_{y \in X} r_{2}(y) y+\sum_{\substack{w \in X \\
z \in X}} t_{2}(z, w) e(z) w \\
p^{k} x \theta=\sum_{y \in X} r_{3}(y) y+\sum_{\substack{w \in X \\
z \in X}} t_{3}(z, w) e(z) w
\end{gathered}
$$

where there are only a finite number of nonzero terms, and all of the $f$ 's and $r$ 's are elements of $R$.

Substituting all this in the previous equation, we obtain

$$
\begin{aligned}
\sum r_{2}(y) y & +\sum t_{2}(z, w) e(z) w=a(x)\left[\sum r_{1}(y) y+\sum t_{1}(z, w) e(z) w\right] \\
& +b(x)\left[\sum r_{3}(y) y+\sum t_{3}(z, w) e(z) w\right]
\end{aligned}
$$

(Here, and in all that follows, the element $x$ is fixed, and the summation is over the elements $z$ and $w$ of $X$. In every case, you sum over $z$ or $w$ if and only if it appears twice in the given expression.) The elements 1 , $a(x), b(x), a(x) b(z)$, etc. arc elements of $R^{*}$ independent over $S$. By Lemma 6, the coefficients of each of these terms in the above equation $\mathrm{m}$ ust be zero. Immediately we see that $\Sigma r_{2}(y) y=0$, and since the elements $y$ are independent over $R$, we obtain

$$
r_{2}(y)=0, y \in X
$$

Looking at the coefficient of $a(x) a(z)$ (for all $z$ ) we see that $\sum t_{1}(z, w) w=0$, whence

$$
t_{1}(z, w)=0, z \in X, w \in X
$$

Looking at the coefficient of $b(x) a(z), z \neq x$, we obtain

$$
t_{3}(z, w)=0,(z \neq x, z \in X, w \in X) \text {. }
$$

From the coefficient of $a(z), z \neq x$, we obtain

$$
t_{2}(z, w)=0,(z \neq x, z \in X, w \in X),
$$


and the coefficient of $a(x)$ gives us

$$
r_{1}(y)=t_{2}(x, y),(y \in X) .
$$

The equations (1), (2) and (3) now become (using (5)-(9)),

$$
\begin{gathered}
p^{k}(1 \theta)=\Sigma r_{1}(y) y \\
p^{k} e(x) \theta=\Sigma r_{1}(y) e(x) y \\
p^{k} x \theta=\Sigma r_{3}(y) y+\Sigma t_{3}(x, w) e(x) w .
\end{gathered}
$$

Now expand $p^{k} e(x) \theta$, using the definition and $\left(1^{\prime}\right)$ and $\left(3^{\prime}\right)$, to obtain

$$
\begin{aligned}
p^{k} e(x) \theta= & p^{k}[a(x)(1 \theta)+b(x)(x \theta)]=a(x) \sum r_{1}(y) y+b(x) \sum r_{3}(y) y \\
& +b(x) a(x) \Sigma t_{3}(x, w) w+b(x)^{2} \Sigma t_{3}(x, w) x w .
\end{aligned}
$$

Setting this equal to $\left(2^{\prime}\right)$, and using Lemma 6 again, we obtain from the coefficient of $b(x)$

$$
\Sigma r_{1}(y) x y=\Sigma r_{3}(y) y
$$

and from the coefficient of $b(x) a(x)$

$$
\Sigma t_{3}(x, w) w=0
$$

Substituting these in $\left(3^{\prime}\right)$, and comparing with $\left(1^{\prime}\right)$, we obtain

$$
x(1 \theta)=x \theta
$$

This shows that the endomorphism $\theta$ is given by right multiplication by an element $(1 \theta)$ of $A$. Hence, $A$ is exactly the endomorphism ring of $M$, as required.

\section{REFERENCES}

1. R. Baer, The decomposition of enumerable, primary, Abelian groups into direct summands, Quart. J. Math., 6 (1935), 217-221.

2. S. Brenner, Modular representations of p-groups, J. Algebra, 15 (1970), 89-102.

3. - Some modules with nearly prescribed endomorphism rings, J. Algebra, 23 (1972), $250-262$.

4. R. R. Colby, On indecomposable modules over rings with minimum condition, Pacific J. Math., 19 (1966), 23-33.

5. A. L. S. Corner, Every countable torsion-free ring is an endomorphism ring, Proc. London Math. Soc., 13 (1963), 687-710. 
6. A. L. S. Corner and P. Crawley, An Abelian p-group without the isomorphic refinement property, Bull. Amer. Math. Soc., 75 (1968), 743-745.

7. L. Fuchs, Infinite Abelian Groups, 2 vol., Academic Press, New York, 1970 and 1973.

8. P. Griffith, On the decomposition of modules and generalized left uniserial rings, Math. Ann., 184 (1970), 300-308.

9. I. Kaplansky, Projective modules, Ann. Math., 68 (1958), 373-377.

10. C. M. Ringel and H Tachikawa, QF-3 rings, to appear in J. Reine Angew. Math.

11. A. V. Roiter, Unboundedness of the dimensions of indecomposable representations of algebras having infinitely many indecomposable representations, Izvestija Akad. Nauk S.S.S.R. Ser. Math., 32 (1968), 1275-1282.

12. R. B. Warfield, Jr., Decomposability of finitely presented modules, Proc. Amer. Math. Soc., 25 (1970), 167-172.

13. - Rings whose modules have nice decompositions, Math. Z. 125 (1972), 187-192.

Received May 30, 1974.

UNIVERSITY OF WASHINGTON 


\section{PACIFIC JOURNAL OF MATHEMATICS}

\section{EDITORS}

RICHARD ARENS (Managing Editor)

University of California

Los Angeles, California 90024

\author{
R. A. Beaumont \\ University of Washington \\ Seattle, Washington 98105
}

\section{J. DugundII}

Department of Mathematics

University of Southern California

Los Angeles, California 90007

D. Gilbarg and J. Milgram

Stanford University

Stanford, California 94305

\section{ASSOCIATE EDITORS}
E. F. BECKENBACH
B. H. NeumanN
F. WoLF
K. YoshidA

\section{SUPPORTING INSTITUTIONS}

\author{
UNIVERSITY OF BRITISH COLUMBIA \\ CALIFORNIA INSTITUTE OF TECHNOLOGY \\ UNIVERSITY OF CALIFORNIA \\ MONTANA STATE UNIVERSITY \\ UNIVERSITY OF NEVADA \\ NEW MEXICO STATE UNIVERSITY \\ OREGON STATE UNIVERSITY \\ UNIVERSITY OF OREGON \\ OSAKA UNIVERSITY
}

\author{
UNIVERSITY OF SOUTHERN CALIFORNIA \\ STANFORD UNIVERSITY \\ UNIVERSITY OF TOKYO \\ UNIVERSITY OF UTAH \\ WASHINGTON STATE UNIVERSITY \\ UNIVERSITY OF WASHINGTON \\ AMERICAN MATHEMATICAL SOCIETY
}

The Supporting Institutions listed above contribute to the cost of publication of this Journal, but they are not owners or publishers and have no responsibility for its contents or policies.

Mathematical papers intended for publication in the Pacific Journal of Mathematics should be in typed form or offset-reproduced (not dittoed), double spaced with large margins. Underline Greek letters in red, German in green, and script in blue. The first paragraph or two must be capable of being used separately as a synopsis of the entire paper. Items of the bibliography should not be cited there unless absolutely necessary, in which case they must be identified by author and Journal, rather than by item number. Manuscripts, in duplicate, may be sent to any one of the four editors. Please classify according to the scheme of Math. Reviews, Index to Vol. 39. All other communications should be addressed to the managing editor, or Elaine Barth, University of California, Los Angeles, California, 90024.

100 reprints are provided free for each article, only if page charges have been substantially paid. Additional copies may be obtained at cost in multiples of 50.

The Pacific Journal of Mathematics is issued monthly as of January 1966. Regular subscription rate: $\$ 72.00$ a year (6 Vols., 12 issues). Special rate: $\$ 36.00$ a year to individual members of supporting institutions.

Subscriptions, orders for back numbers, and changes of address should be sent to Pacific Journal of Mathematics, 103 Highland Boulevard, Berkeley, California, 94708.

PUBLISHED BY PACIFIC JOURNAL OF MATHEMATICS, A NON-PROFIT CORPORATION Printed at Jerusalem Academic Press, POB 2390, Jerusalem, Israel.

\section{Copyright (C) 1975 Pacific Journal of Mathematics All Rights Reserved}




\section{Pacific Journal of Mathematics

Vol. 60, No. $2 \quad$ October, 1975

Waleed A. Al-Salam and A. Verma, A fractional Leibniz q-formula ........... 1

Robert A. Bekes, Algebraically irreducible representations of $L_{1}(G) \ldots \ldots \ldots \ldots 11$

Thomas Theodore Bowman, Construction functors for topological

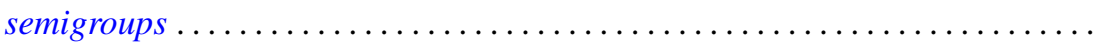

Stephen LaVern Campbell, Operator-valued inner functions analytic on the

closed disc. II .........................................

Leonard Eliezer Dor and Edward Wilfred Odell, Jr., Monotone bases in $L_{p} \ldots \ldots$.

Yukiyoshi Ebihara, Mitsuhiro Nakao and Tokumori Nanbu, On the existence of

global classical solution of initial-boundary value problem for

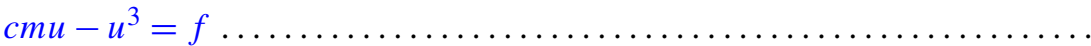

Y. Gordon, Unconditional Schauder decompositions of normed ideals of

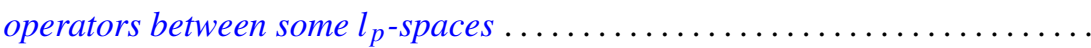

Gary Grefsrud, Oscillatory properties of solutions of certain nth order functional

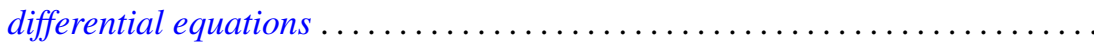

Irvin Roy Hentzel, Generalized right alternative rings ...................

Zensiro Goseki and Thomas Benny Rushing, Embeddings of shape classes of compacta in the trivial range .................................

Emil Grosswald, Brownian motion and sets of multiplicity . .

Donald LaTorre, A construction of the idempotent-separating congruences on a

bisimple orthodox semigroup .

Pjek-Hwee Lee, On subrings of rings with involution ...

Marvin David Marcus and H. Minc, On two theorems of Frobenius ...

Michael Douglas Miller, On the lattice of normal subgroups of a direct

product. .

Grattan Patrick Murphy, A metric basis characterization of Euclidean space

Roy Martin Rakestraw, A representation theorem for real convex functions ....

Louis Jackson Ratliff, Jr., On Rees localities and $H_{i}$-local rings ...

Simeon Reich, Fixed point iterations of nonexpansive mapping . .

Domenico Rosa, $B$-complete and $B_{r}$-complete topological algebras ...

Walter Roth, Uniform approximation by elements of a cone of real-valued

functions ....

Helmut R. Salzmann, Homogene kompakte projektive Ebenen

Jerrold Norman Siegel, On a space between $B H$ and $B_{\infty} \ldots$

235

Robert C. Sine, On local uniform mean convergence for Markov operators

James D. Stafney, Set approximation by lemniscates and the spectrum of an

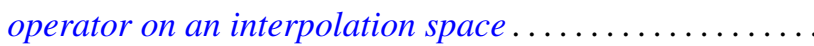

Árpád Száz, Convolution multipliers and distributions .......

Kalathoor Varadarajan, Span and stably trivial bundles ..........

Robert Breckenridge Warfield, Jr., Countably generated modules over

commutative Artinian rings....................... 